\title{
Design and testing of PhotoDetector covers for chain implosion prevention in Hyper-KamiokaNDE
}

\author{
David Bravo-Berguño*† \\ Universidad Autónoma de Madrid, Campus de Cantoblanco - 28049 Madrid \\ E-mail: david.bravob@uam.es
}

\begin{abstract}
The Hyper-Kamiokande (HK) experiment aims to address the most fundamental questions of particle physics today through its design as a multi-purpose water-Čerenkov detector with a $40 \%$ photocathode coverage. Proper safeguards must be taken against improbable, but catastrophic events such as a chain implosion of thousands of photomultiplier tubes (PMTs). Here, we present the evolution and testing results of the stainless-steel UAM-Aratz HK PMT cover, also known under the informal designation of "Spanish cover". The design, modelization and testing phases carried out during 2017 and early 2018 have shown it to be a reliable low-cost alternative for large-scale PMT implosion protection in HK. Follow-on work will focus on weight reduction while maintaining its robustness and economicity.
\end{abstract}

XIV International Conference on Heavy Quarks and Leptons (HQL2018)

May 27- June 1, 2018

Yamagata Terrsa, Yamagata,Japan

\footnotetext{
* Speaker.

${ }^{\dagger}$ On behalf of the Hyper-Kamiokande Collaboration. Also at INFN Milano (Italy).
} 


\section{Introduction: Hyper-Kamiokande and the need for PMT covers}

The Hyper-Kamiokande (HK) experiment is formulated as a multi-purpose ultra-pure waterČerenkov ring imaging detector aimed at shedding light on the most crucial present questions in particle physics[1]. It is expected to start operations in the mid-2020s. Through its sensitivity to neutrinos, both natural (supernova burst and relic, atmospheric, solar, geological, astrophysical...) and man-made (produced in the Tokai accelerator as part of the T2HK initiative), as well as to nucleon decay and WIMP dark matter, HK is expected to provide groundbreaking insights into matterantimatter asymmetry $\left(\delta_{C P}\right)$, neutrino mixing $\left(\theta_{23}\right.$ maximality and octant, mass ordering and absolute scale, MSW precision tests...), Non-Standard Interactions (NSI) and Beyond-Standard Model (BSM) neutrino physics (sterile or heavy neutrinos, CPT/Lorentz invariance tests...), and astro- and heliophysics (black hole formation, core infall processes in gravitational wave generation, heavy element production, solar core temperature variability, solar composition problem...).

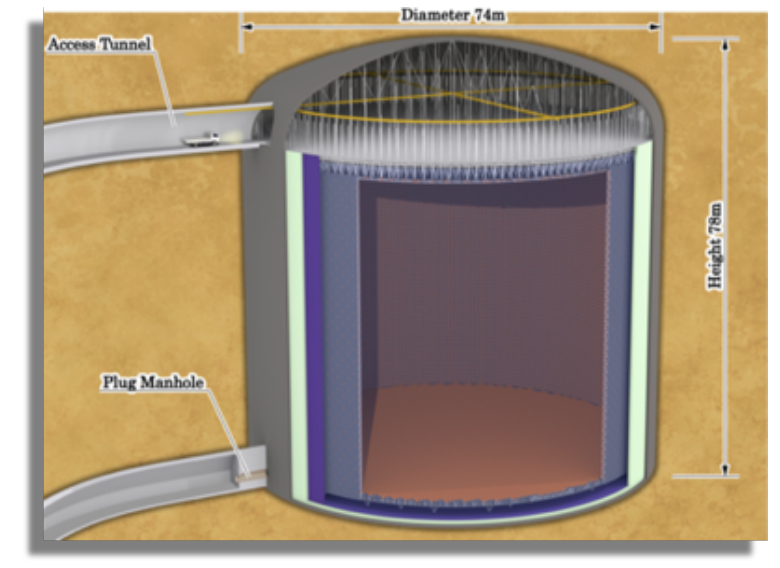

Figure 1: Hyper-Kamiokande detector conceptual artist's rendering.

The HK detector will consist of a cylindrical water tank $(60 \mathrm{x} 60 \mathrm{~m})$ excavated $\sim 8 \mathrm{~km}$ away from the current Super-Kamiokande (SK) detector, as illustrated in Figure 1. It will be instrumented by tens of thousands of R12860-HQE photomultiplier tubes (PTMs) offering a 40\% photocathode coverage in its Inner Detector, which will also be surrounded by a more sparsely-covered Outer Detector for background vetoing (using R5912 PMTs).

As illustrated by the November 12th, 2001 chain implosion of 6665 ID and 1027 OD PMTs in the SK detector[2], a single minor, statistically-infrequent defect in a PMT can have catastrophic consequences due to the hydraulic hammering effect caused by an eventual shockwave stemming from the implosion of the photosensor's glass vacuum enclosure. In SK, the PMTs were protected starting in the SK-II and SK-III phases with fiberglass-reinforced plastic (FRP) covers - but this design was seen to have room for optimization, both in cost and intrinsic radioactive background.

For HK, the R12860 design will offer several photodetection improvements[1], as well as superior mechanical strength in the fragile neck area through an optimized glass bulb shape. However, it is desired to complement this intrinsic improvement with an easily manufacturable, cost-effective and resistant photodetector cover to minimize chain implosions risks as much as possible. 


\section{PMT cover designs}

Three complementary designs are currently being tested: a stainless-steel conical cover (also known as the "Japanese SUS cover"), a resin conformal cover (a.k.a. "Japanese resin cover") and a stainless-steel pseudo-cylindrical cover (a.k.a. "Spanish cover"). All models allow for pressure equalization, with water filling the space between PMT and cover interior. A PMT implosion creates a partial vacuum that slowly fills in, preventing a strong shockwave and its transmission.

The "Spanish" cover has seen three major design iterations, whose characteristics are detailed below. All of them use the same structural material (AISI 304 stainless steel) and are based on a sheet thickness of 2 to $2.5 \mathrm{~mm}$, depending on the location of the piece. The design is the fruit of the collaboration of the Madrid Autonomous University (UAM) with Talleres Aratz S.A. of Vitoria, Spain, and is focused on lowering costs, both in manufacturing and transportation (only 2-3 individual pieces), as well as simplified provisions for PMT mounting. All covers are designed to be joined to a Kuraray Co. (Japan) transparent acrylic cover on their upper side (15 or 20 $\mathrm{mm}$ ), through which the PMT can intake photons and retain most of its optical acceptance. Eight stainless-steel threaded rods ("gap spanners") firmly affix the PMT on the sides for positioning.

Prototype units for testing have all been handcrafted, since the overhead costs for high-precision serial-production tooling are too high for a few unique parts. This strategy has raised per-unit production costs and worsened tolerances, as expected - but also proven adequate for design validation.

\subsection{Version 1}

This design consists of a cylindrical "barrel" section made up of two semicylindrical sections ("tejas") which are bolted together at a straight, radially-protruding flange (see Figure 2). A stretched hemispherical dome covers the back side and is bolted to the cylindrical walls. This dome is fabricated through an embossing technique for all prototype versions. Finally, the front acrylic dome is bolted to a flange bent outward from the cylindrical pieces. The weight of the $\mathrm{v} 1$ design was $21 \mathrm{~kg}$. Version 1 has seen two test iterations, informally referred to as v1.0 and v1.1, which incorporated reinforcements. Cover v1.1 was never produced, being substituted by Version 2.

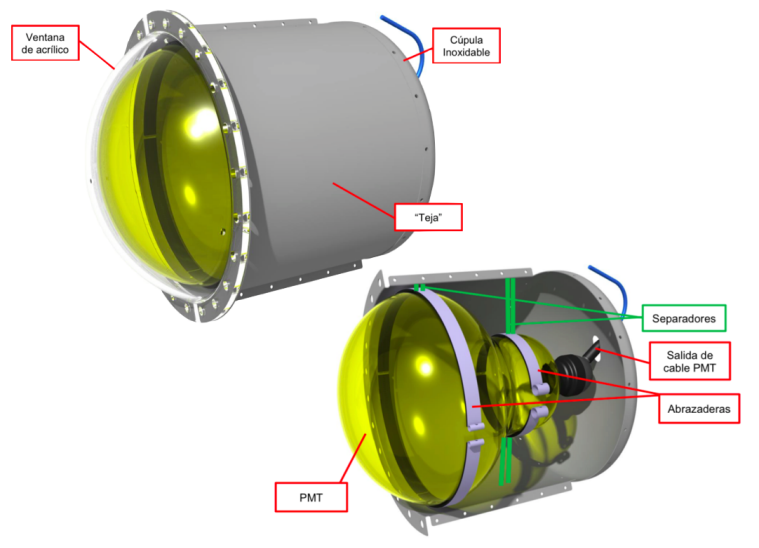

Figure 2: Labelled v1.0 cover: on the exterior view, the "acrylic window" (left), stainless-steel dome (upper right) and sidewall ("teja", lower right); on the cutout view, gap-spanners (upper right), PMT cable exit hole (center right) and PMT bands (lower right). 


\subsection{Version 2}

This design replaces the simple cylindrical section to a single-piece welded truncated-conical wall section (see Figure 3's left section). The bottom endcap is now longer, and its interface with the wall section inside the wall piece. The top flange, to which the acrylic is bolted, is replaced by a screw-on, separate, very thick ring flange intended to qualify the main cover design under the acrylic dome. It provides most of the strength on the top, but also the weight (adds $10.5 \mathrm{~kg}$ ). The complete v2 cover design weighs $30.7 \mathrm{~kg}$. Version 2 has seen three model iterations informally referred to as v2.0, v2.1 and the latest version, v2.2 (which successfully passed all testing so far)

\subsection{Version 3}

This design retains the same general characteristics as v2.2, but is envisioned to lighten it by $>10 \mathrm{~kg}$ by removing the ring flange and attaching a "flangeless" Kuraray acrylic cover directly to the wall section (see Figure 3's right section). This version is still in its final design phase, although performance has been preliminarily assessed through Finite Element Model (FEM) analyses.
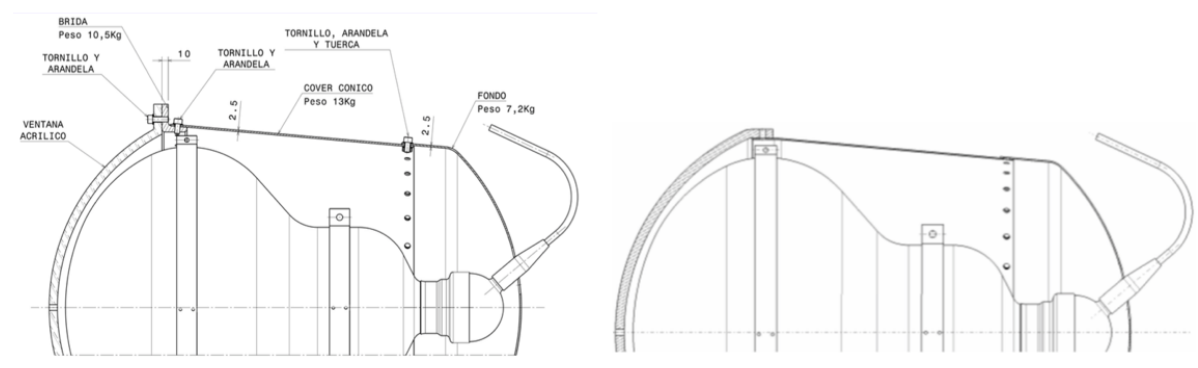

Figure 3: Technical drawings of a v2.0 cover (left) and the concept of a v3 type (right).

\section{UAM-Aratz ("Spanish") cover testing results}

\subsection{FEM analysis}

Initial testing focused on (i) structural performance under buoyancy (upward $60 \mathrm{~kg}$ force through the PMT-to-cover attachment points via the gap spanners, i.e. the regime under nominal conditions) and (ii) implosion performance (constant, homogeneous pressure of $0.9 \mathrm{MPa}$ on all exterior surfaces of the cover+acrylic system, simulating a hydrostatic pressure test). After low stresses were seen for the v1 setup in (i), it was not deemed necessary to repeat the test for similar refined iterations ( $\mathrm{v} 2$ and $\mathrm{v} 3$ ), and only (ii) was performed for these evolved designs.

- Version 1. A v1.0 model was developed for which four points were considered fixed, and therefore allowed no displacement due to the forces exerted on the cover. Low stresses of up to $55 \mathrm{MPa}$ at the gap spanner bolting points, and displacements of under $0.07 \mathrm{~mm}$, were seen on the buoyancy regime simulations. For the implosion regime, tests results appeared positive, although the presence of small high pressure pressure point-like areas on scenario (ii) needed further investigation with real-world testing. 
- Version 2. The results showed improved displacement results of under $0.5 \mathrm{~mm}$ and maximum widespread stresses of $\approx 400 \mathrm{MPa}$, with peaks under $700 \mathrm{MPa}$, as seen in Figure 4. It was also determined the design would fail at around $\approx 1.0 \mathrm{MPa}$.

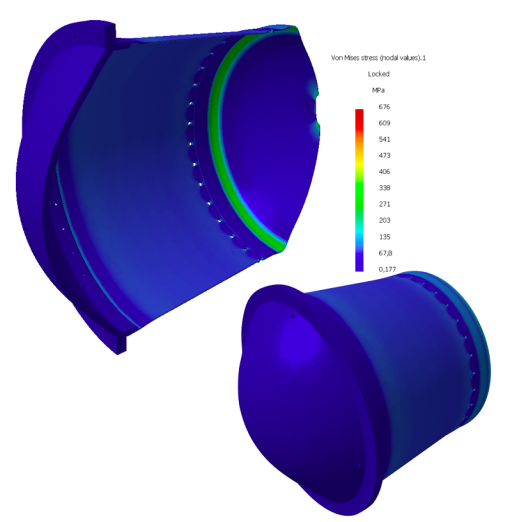

Figure 4: Stress results for the FEM of the v2.0 design subject to 0.9 MPa of external pressure.

- Version 3. Results showed maximum stresses below $450 \mathrm{MPa}$. These maximally-stressed points now lie at the acrylic-to-wall bonding interface, concentrated around the bolt holes where the structure is fixed and not allowed to move - and are therefore expected to be better protected against deformations by the double layer of material around them. More widespread maximum stresses were roughly $60 \%$ of those typical in the v1 design.

\subsection{Hydrostatic testing}

Real-world analysis was performed in two stages: firstly hydrostatic testing, involving wrapping the empty cover prototypes in water-tight plastic sheets (to avoid their interior flooding and keep the pressure differential), to then apply pressure on them inside a closed container. The second campaign was implosion testing, where a PMT glass bulb (without electrodes) was used as the specimen to be imploded inside the cover. Several other PMT glass bulbs were positioned around the cover in order to demonstrate shockwave attenuation at different distances and positions.

- Version 1. Four hydrostatic tests were performed in Kamioka with three v1.0 cover prototypes and $15 \mathrm{~mm}$ acrylic dome. In all test articles, tolerances between the endcap and wall sections were quite large, resulting in a loose endcap compared to the wall cylindrical diameter. As a result, the pieces were pretensioned with large stresses - causing buckling and failure. The test results and their dissonance with the expected pressure tolerance evidenced the need for improvements, especially at the endcap-to-wall interface, before attempting implosion tests - prompting the re-design to v1.1, and eventually v2.

- Version 2. Three hydrostatic tests were performed in Vitoria (Spain), in a dedicated pressure chamber derived from water reservoir high-pressure injectors also fabricated at the site of Talleres Aratz S.A. This test series demonstrated the company's rapid diagnostics and repair turnaround to an unexpected defect that would only manifest itself in prototype handcrafted articles. Through the interim v2.1, separate failure modes were isolated. A solid v2.2 design was then successfully tested up to $0.7 \mathrm{MPa}$ with no damage. 


\subsection{Implosion testing}

Only the v2.2 design, which had successfully proven its worth during hydrostatic testing, was implosion-tested. Implosion tests for the three cover models ("Japanese SUS", "Resin" and "Spanish v2.2") were performed in the ex-JApanese MIcrogravity Center (JAMIC) facilities in Kami-Sunagawa (Hokkaido, Japan) in early March 2018. This facility offers a $\approx 700$ m-deep shaft filled with water at a constant $\approx 12^{\circ} \mathrm{C}$ temperature[3]. One "Spanish" cover was built on-site and paired with a $20 \mathrm{~mm}$-thick Kuraray acrylic dome. Six strain gauges were stuck on the outer surfaces of the cover and acrylic dome. A chemically-triggered device with a metal conical termination that impacted the PMT neck ("pusher") protruded into a hole in the cover towards the PMT surface in order to trigger its collapse at the desired moment. The cover, holder and pusher ensemble was rigged in a larger metal frame with 4 "witness" bare PMTs around it. Pressure gauges were also installed on the rig around the cover to assess the pressure environment just after the implosion.

The test was carried out at a depth of 60 meters, and saw a successful PMT implosion, albeit with a slightly delayed dome collapse compared to most implosions of this kind. However, pressure gauge results showed a satisfactory overpressure shockwave was achieved $(\approx \pm 0.2$ Mpa upon PMT implosion). The acrylic's maximum strain was about $\mu \approx-1500$, while the cover body saw strains of $\mu \approx-70$ (with peaks of $\mu \approx \pm 500$ ). The cover successfully prevented a chain implosion with no apparent damage (see Figure 5).

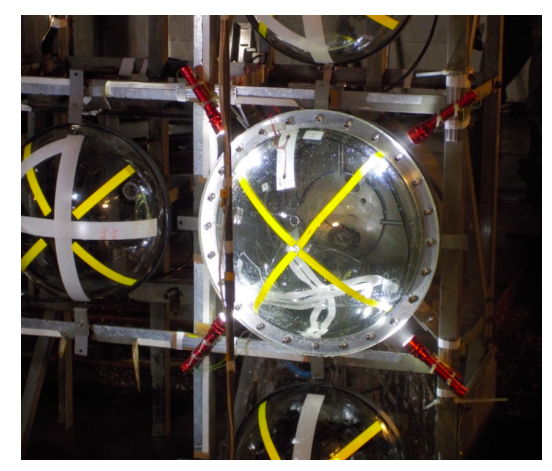

Figure 5: Cover photograph just after emergence from the test pit. Note the surviving surrounding PMTs and the shards of the imploded PMT inside the cover.

\section{Conclusions and future perspectives}

The v2.2 "Spanish" cover design withstood all FEM, hydrostatic and implosion testing, with no apparent damage, either to the SUS structure itself or the acrylic dome. Surrounding bare PMTs were unaffected by the implosion, thereby validating the UAM-Aratz v2.2 cover design at up to 60 $m$ depth. It is however difficult to establish a direct comparison with the other HK cover designs: fundamentally, no other cover was tested successfully at a similar depth. The acrylic was $5 \mathrm{~mm}$ thicker than those used for the other covers (a secondary objective of the test was to validate it), and the thicker dome would make the ensemble more resistant. The recorded video, being pointed directly at the PMT dome, also could not unequivocally prove whether the PMT implosion was equivalent to previous ones. For these reasons, further v2.x and v3 tests are desirable. 


\section{Acknowledgements}

This research has been directly funded by funds from the European Union H2020-MSC RISEGA641540-SKPLUS and COFUND2015-GA713366-InterTalentum (NUOSCILLATION), as well as the support of the Hyper-Kamiokande proto-Collaboration.

\section{References}

[1] Hyper-Kamiokande proto-Collaboration (K. Abe et al.) Hyper-Kamiokande Design Report arXiv-physics.ins-det:1805.04163

[2] Chiaki Yanagisawa for the SK Collaboration Report on the Super-Kamiokande Accident II - retrieved from superk.physics.sunysb.edunngrouppublication_thesesSKaccident02.ps

[3] A. Koide JAMIC Drop-shaft Type Microgravity Facility Accommodating 10-seconds Microgravity Journal of Japan Microgravity Application Society, Vol. 18 No. 32001 (136-139) 